\title{
Sentinel Lymphadenectomy - Ready for Clinical Routine?
}

One of the most important principles of current strategies in mostly all medical fields is the individualization of therapy guided by the individual needs of each patient. Beside new techniques for molecular diagnostics, the sentinel lymph node biopsy (SLNB) is one of the approaches to the individualization of surgical therapy and, moreover, to individualized multimodality treatment. The node that has the highest probability of tumor infiltration and, thus, reflects the nodal status of the adjacent lymph node region is called the sentinel lymph node (SLN). I nstead of a systematic lymph node dissection a selective SLNB reveals the nodal status of the lymph node region. The major goal is the selection of patients with a tumor-infiltrated SLN for lymph node dissection of the corresponding lymph node region, whereas in nodal-negative patients, trauma, costs and morbidity of the dissection can be avoided.

A fter 7 years of continuous, controlled evaluation it has become obvious that the method is a safe and practicable way to determine the axillary nodal status in patients with small breast cancer and clinically negative lymph nodes. D etection rates of more than $90 \%$ and an accuracy in the prediction of the nodal status of $95-100 \%$, controlled by a complete axillary lymph node dissection (A LND) subsequent to SLNB, were described after a relatively short period of training [1-3]. Moreover, after selective histopathologic evaluation using more serial sections and immunohistochemical staining, the rate of nodal-positive patients could be increased by the detection of micrometastases in about $10 \%$ of the cases [4].

The convincing results have led to widespread routine use of the SLNB in experienced centers of the U SA [5], whereas in Europe, large, randomized, long-term controlled studies to compare the results of SL NB with complete systematic dissection are currently undertaken or still in preparation. A ims of these studies are:

(1) to prove that, even in clinical practice, the false-negative rate (rate of patients falsely determined as nodal-negative) of the SL N B does not lead to a worse clinical outcome;

(2) to prove that the morbidity after SLNB in nodal-negative patients is lower than in patients with complete node dissection (A LND).

H owever, if we are aware of more than 100 published studies demonstrating excellent detection rates and a very high accu- racy in predicting the nodal status, controlled by $A$ LND subsequent to $S L N B$, the question must be raised: What do we really want to know now? We feel that there are questions left to answer, but there are no further queries if the method works or if it is somewhat 'good'. In contrast, the discussion should focus on the following:

First, after all these studies with varying techniques and different patient subgroups we have to evaluate the optimal method for the optimal patient. This means that reasonable standards have to be introduced based on the results of the published studies, both for the radionuclide and for the blue dye method. In randomized studies it must be further clarified which details of each method should be changed or optimized, for example, which injection technique (intraparenchymal, subdermal, subareolar) is to prefer for the individual patient, etc.

Second, there must be a quality control for surgeons and centers applying the method in a therapeutic concept. A pproved training centers should guarantee a sufficient formation and training for the performing surgeons, and each center should undergo controls of the results of SLNB (evaluated by subsequent A LND) and the logistic conditions in the hospital until the certification is approved.

Third, the impact of micrometastases in the SL N detected by $\mathrm{HE}$ staining ( $<2 \mathrm{~mm}$ in diameter) as well as micrometastases detected by immunohistochemistry has to be evaluated in regard to its impact on local failure and prognostic significance and in regard to its impact on the subsequent therapy.

Concerning morbidity, it seems to be very obvious that a selective biopsy of one or two lymph nodes should cause significant less morbidity than complete A LND. Moreover, Schrenk et al. [6] showed most recently in a randomized study of 70 patients, using a combination of the blue dye and the radionuclide method, that the morbidity after SLNB is negligible and the reduction of morbidity in comparison to A L ND is significant.

In conclusion, we feel that the SLNB is an important step to surgical individualization of cancer therapy. A s for melanoma patients, the method is of major concern for early breast cancer patients. It is now the time for experienced centers to introduce this method into clinical practice for the benefit of

\begin{tabular}{ll}
\hline KARGER & @ 2000 S. K arger G mbH , Freiburg \\
Fax +497614520714 & A ccessible online at: \\
$\begin{array}{l}\text { E-mail Information@K arger.de } \\
\text { www.karger.com }\end{array}$ & www.karger.com/journals/onk
\end{tabular}


early-stage breast cancer patients in E urope. N evertheless, it is necessary to define exactly the technical standards and to introduce an approval certificate of quality requirements and quality controls for performing centers. Randomized studies comparing the feasibility and the accuracy of SLNB with standard A LND in centers that have already reached a high level of performance do not seem to provide new information, but they withdraw patients of the A LND group from the benefit of SLNB.

A. B embenek, P.M. Schlag, B erlin

\section{References}

1 B embenek A, Reuhl T, M arkwardt J, Schneider U, Schlag PM : Sentinel lymph node dissection in breast cancer. Swiss Surg 1999:5:217-221.

$2 \mathrm{~K}$ rag $D N$, Weaver $D L$, A shikaga $T, M$ offat $F, K$ lim berg V S, Shriver C, Feldman S, Kusminsky R, G add $M$, Kuhn J, H arlow S, B eitsch P: The sentinel node in breast cancer. N Engl J M ed 1998;339:941-946.
3 Schlag PM, Veronesi U : Lymphatic M etastasis and Sentinal Node Biopsy. Recent Results in Cancer R esearch, vol 157. B erlin, Springer, 2000.

4 Schreiber R H, Pendas S, Ku N N, R eintgen DS, Shons A R, B erman C, B oulware D, Cox CE : M icrostaging of breast cancer patients using cytokeratin staining of the sentinel lymph node. Ann Surg Oncol 1999; 6:95-101.
5 Cox CE, Pendas S, Cox JM, Joseph E, Shons A R Yeatman T, Ku N N, Lyman GH, Berman C, H addad $F$, R eintgen DS: Guidelines for sentinel node biopsy and lymphatic mapping of patients with breast cancer. A nn Surg 1998;227:645-653.

6 Schrenk P, Rieger R, Shamiyeh A, Wayand W Morbidity following sentinel lymph node biopsy versus axillary lymph node dissection for patients with breast carcinoma. Cancer 2000;88:608-614. 